\title{
LA CONCIENCIA CONTENIDA. ANÁLISIS DEL PROBLEMA DE LA CONCIENCIA EN LA HIPÓTESIS DE LA MENTE EXTENDIDA DE ANDY CLARK Y DAVID CHALMERS
}

\author{
Óscar Barea Manuel \\ Universidad de Murcia \\ newbarea@gmail.com
}

\section{RESUMEN}

El objetivo de este trabajo es, tras haber esbozado la noción de mente extendida propuesta originalmente por Andy Clark y David Chalmers en su artículo «The extended mind» (1998), exponer los argumentos que respaldan la creencia de una cognición extendida, así como las visiones que se presentan críticas a esta postura. Tras ello nos introduciremos en el eje central del ensayo, que versa sobre los argumentos a favor y en contra de la posibilidad de una conciencia extendida fuera del sistema nervioso central.

Palabras clave: mente extendida, funcionalismo, externalismo, conciencia, cognición.

\author{
THE CONTAINED CONCIOUSNESS. ANALYSIS OF THE PROBLEM \\ OF CONSCIOUSNESS IN THE ANDY CLARK AND DAVID CHALMERS'S \\ EXTENDED MIND HYPOTHESIS
}

\section{Abstract}

The aim of this work is, after having outlined the notion of extended mind originally proposed by Andy Clark and David Chalmers in their article «The extended mind» (1998), to expose the arguments that support the belief of extended cognition, as well as the critics of this position. After that, we will enter to the main axis of the essay, which deals with the arguments for and against the possibility of an extended consciousness outside the central nervous system.

KEYwORDs: extended mind, functionalism, externalism, consciousness, cognition. 


\section{LOS ARGUMENTOS DE LA TESIS DE LA MENTE EXTENDIDA}

«¿Dónde termina la mente y comienza el resto del mundo?»" Con esta pregunta abre el artículo publicado por Andy Clark y David Chalmers, filósofos de la mente y catedráticos de la Universidad de Edimburgo y la Universidad Nacional de Australia, respectivamente. Según su postura, los límites no están tan claros como hasta el momento se ha intentado afirmar en las distintas hipótesis acerca del problema de la relación entre mente y cuerpo. La mente no parece hallarse en un lugar delimitado y evidente, como puede ser el sistema nervioso central o, más vagamente, el cerebro. Esta última visión sería la defendida por el internismo, donde los límites de la mente son establecidos por la piel y el cráneo.

En oposición a los planteamientos epistemológicos del internismo, Clark y Chalmers defienden un externismo activo, donde el entorno juega un papel fundamental en la ejecución de los procesos cognitivos, en la medida en que el sujeto tiende a apoyarse en éste de manera bastante considerable. No obstante, resulta necesario diferenciar la tesis del externismo activo frente al llamado externismo de contenido, como el que defienden Burge o Putnam ${ }^{2}$. A tal efecto, debemos destacar varios puntos del ejemplo de la Tierra Gemela, ideado por Putnam para ilustrar su propuesta teórica. En primer lugar, el planteamiento de Putnam versa sobre los contenidos de los estados mentales, mientras que en la tesis de la mente extendida (en adelante, TME) el eje central será la cuestión de los vehículos de la cognición. Así pues, Putnam o Burge defienden que los contenidos mentales de seres idénticos molécula a molécula serían distintos en dos mundos posibles, pues ese contenido mental es individuado externistamente por las diversas relaciones entre el individuo y el entorno, tanto por la distinta composición natural de ambos contextos como por las diferentes prácticas de las comunidades lingüístico-conceptuales.

Otro elemento para destacar es que este externismo de contenido genera problemas, siendo el más considerable que dicho planteamiento no parece muy condescendiente con el estudio científico de la mente y la conducta humana. Esto es así porque cualquier propuesta científica rigurosa que se precie, debe dar una explicación causal plausible de la conducta con tipos unitarios. Si hablamos de un contenido mental que se individúa externistamente, dichas explicaciones causales variarán con todos los contextos posibles; y en ese caso sería imposible tratar de hacer ciencia en sentido estricto, pues el resultado - que sería, al fin y al cabo, un determinado contenido mental- siempre sería variable dado uno u otro contexto.

La opción más plausible para resolver el dilema de incompatibilidad entre ciencia cognitiva y externismo sería aceptar que los distintos tipos intencionales que aparecen en las explicaciones causales científicas se individúan internistamente, en

nes, p. 61.

${ }^{1}$ Clark, A. y Chalmers, D. (2011). La mente extendida. Oviedo, España: KRK Edicio2 Ibid., pp. 63-67. 
lugar de externistamente. Así, podrían darse casos predecibles e idénticos entre los dos individuos idénticos en los distintos contextos de la Tierra y la Tierra Gemela. La explicación común entre ambos individuos, que generaría similitudes aunque de súbito fuesen intercambiados contextualmente, es explicada por tipos intencionales individuados internistamente.

Aceptando esta opción, caben dos tipos de contenido mental compatibles entre sí: el contenido mental amplio y el contenido mental estrecho. Todo estado mental puede tener este doble contenido. El primero refiere a un tipo de contenido mental que es individuado externistamente, es decir, se genera dado el entorno y depende de él. De este modo, Putnam y Burge apoyarían esta visión, pues da cuenta de las diferencias de contenido en distintos contextos. El segundo tipo de contenido mental es más afín a la explicación científica de la conducta, pues defiende un contenido no individuado externistamente, no dependiente del contexto. La cuestión es que ese contenido mental estrecho, intuitivamente, tiene que implementarse en estructuras neuronales, pues se trata de algo independiente del entorno; y los vehículos materiales comunes de los individuos idénticos son ésos.

Dicha conclusión es la que niegan Clark y Chalmers. Ambos aceptan el tipo de contenido mental estrecho, pero no defienden que de ahí se siga que los vehículos propios de la cognición sean exclusivamente neuronales. Los contenidos estrechos se pueden implementar en vehículos con componentes parcialmente extraneuronales. Así, aceptan, por un lado, que la explicación científica requiera de contenidos mentales estrechos, pero, por otro, niegan la condena que supone una frontera de los vehículos cognitivos en el cerebro ${ }^{3}$.

Uno de los puntos clave en los que Clark y Chalmers se basan para fundamentar la extensión de la cognición al entorno es la vinculación del organismo humano con el entorno mediante una interacción de doble sentido, logrando así generar lo que llaman un sistema acoplado. Aquí nos hablan de la importancia del papel causal activo de todos los componentes de un sistema, y que si uno de ellos fallase, todo éste se resentiría. El compromiso funcionalista de la TME se ve claramente aquí. El funcionalismo, en su origen durante los años sesenta del pasado siglo, se presentó como una respuesta a los problemas que derivan de la tesis de la identidad, el dualismo y el conductismo lógico. El concepto clave es el de realización múltiple, propuesto por Putnam o Fodor; y la descripción general de la teoría viene a afirmar que los estados mentales son estados funcionales.

Los estados mentales estarían, pues, caracterizados por lo que se conoce como un rol causal, implementado en un sistema neurológico -aunque ese sistema en el que se encuentran implementados podría variar-. La tesis fundamental que presenta Hilary Putnam a lo largo de su obra de 1975, Mind, Language and Reality, es la defensa de la noción de isomorfismo funcional. Dos sistemas son funcionalmente isomórficos cuando se da una correspondencia en los estados de uno y de otro que preserve las relaciones funcionales. Una relación $A \rightarrow B$ en un sistema 1

3 Ibid., pp. 44-49. 
$\left(\mathrm{S}^{1}\right)$ será funcionalmente isomórfica sí y sólo si se da la misma correlación en el sistema $2\left(\mathrm{~S}^{2}\right)$. Así podemos acabar con el problema de la existencia de estados mentales idénticos en diferentes estructuras neurológicas (el problema de las otras especies que mermó las bases de la tesis de la identidad) e incluso no sólo neurológicas, de donde se sigue la cuestión sobre si una computadora podría llegar a poseer eventos mentales en el funcionalismo computacional ${ }^{4}$.

Pero volviendo al punto clave, vemos que la estela funcionalista se hace patente en la TME cuando hablamos de la paridad funcional como defensa principal de la hipótesis de la cognición y la mente extendida. Nótese que hablamos de cognición y mente como conceptos distintos, y en principio los autores los manejan así, puesto que comienzan el artículo hablando de que sólo la cognición está extendida -en el sentido de la facultad de procesar información a partir de la percepción mediante ciertos vehículos-, y más tarde se plantean ampliar a si los propios estados mentales, tales como una creencia o un deseo, son extensibles también.

Por poner un ejemplo que nos permita entender mejor el argumento de la paridad funcional, supongamos que, aunque la memoria biológica de un sujeto es un recurso interno y un bloc de notas para tomar apuntes constituya algo externo, y, evidentemente, hay diferencias muy obvias, no se deben ocultar las enormes similitudes entre ambos recursos, similitudes que son capturables funcionalmente. Por lo tanto, funcionalmente hablando, es decir, atendiendo al rol causal, las creencias de un sujeto que accede a un contenido mental alojado en su memoria y otro que lo hace observando los apuntes de su bloc de notas son isomórficas; es decir, cumplen el mismo rol causal. Y, puesto que acceder al estado mental por parte de la persona que ha utilizado un apunte más allá de su memoria ha exigido de vehículos cognitivos externos a las propias barreras impuestas por la memoria biológica, podríamos hablar de que el propio proceso cognitivo ha requerido de bucles extraneuronales para su implementación. Es por tanto plausible que la cognición exija de componentes de carácter extraneuronal; y de ahí deducimos que la cognición se extienda al entorno.

\section{LOS PROBLEMAS DE UNA PARIDAD FUNCIONAL}

Para poder fundamentar la paridad funcional hay que entender una distinción básica en la que se mueven Clark y Chalmers, y que a su vez se tornará problemática cuando nos introduzcamos en el tema central de la conciencia extendida. Esta distinción es la de similitudes funcionales de grano fino y grano grueso.

Una similitud de grano grueso es capturable mediante una descripción de sentido común, es una semejanza atendiendo al rol causal. Sin embargo, una similitud de grano fino está más centrada en los detalles y sus diferencias. Aunque estas similitudes sí podrían ser capturadas funcionalmente, generarían como resultado unos tipos

\footnotetext{
${ }^{4}$ Putnam, H. (1975). Mind, language and reality. Cambridge, EE. UU.: Cambridge University Press, pp. 291-292.
} 
funcionales que no serían los del sentido común, ya que el grano fino atiende más bien a aquellos circuitos materiales que harían posibles las creencias de los dos sujetos que hemos mencionado. Es por ello por lo que la continua exigencia de basarse en el sentido común para argumentar la cognición extendida lleva sin duda a críticas y contradicciones ${ }^{5}$. Así pues, en este trabajo mostramos la problemática de que una noción de la mente extendida debe lidiar con una enorme tensión. Esta tensión está producida, por un lado, dada la defensa funcionalista de que existen vehículos cognitivos que se fundamentan en el grano grueso (el sentido común), que no dependen de detalles neurológicos, o de lo contrario no tendría sentido hablar de circuitos cognitivos fuera de las limitaciones del sistema nervioso central, y, por otro lado, debido a la defensa de procesos neurológicos en detalle, que explicarían la existencia de la conciencia.

Unos de los principales críticos de la TME son Fred Adams y Ken Aizawa. En su artículo «The bounds of cognition» mantienen una postura que ellos mismos denominan como una forma de intracranealismo contingente, es decir, aunque la ciencia actual haya sugerido que pueda haber casos en los que la cognición se extienda al mundo físico, superando así los límites del propio cuerpo, dichos casos no se han dado fácticamente; aun cabiendo la posibilidad. Su postura es que los objetos del mundo no forman parte de los vehículos de la cognición, y por tanto no existe un sistema cognitivo único formado por el cerebro, el sistema nervioso central, el resto de partes del cuerpo y el entorno.

Para estos dos autores, la defensa de la TME se basa en el sentido común que se da en ciertas situaciones, como un cálculo matemático en un papel, donde el uso de ese material como apoyo parece habilitarnos para superar en cierto modo las limitaciones de las capacidades propias de nuestro cerebro. Adams y Aizawa comparan la propuesta de Clark y Chalmers con la de Daniel Dennett y Merlin Donald. Dennett defiende esa superación de las limitaciones de un cerebro que denomina «animal» y propone un único sistema cognitivo enlazado con el entorno. Merlin, por otro lado, también se une a esta interpretación de una cognición extendida, hasta tal punto que afirma el uso de un «almacén de símbolos externos» como la pieza fundamental y el más dramático paso evolutivo en la cognición humana desde el Australopithecus al Homo sapiens sapiens.

La pretensión de Adams y Aizawa es generar una explicación de sentido común basándose en los conocimientos empíricos de los que disponemos actualmente. Así, tal y como ellos bien afirman, son incomparables los procesos psicológicos que se generan en el cerebro con otros procesos físicos en el entorno. Son de un estatus totalmente distinto, y equipararlos sería como comparar el funcionamiento de un brazo con el de unas tijeras de podar; colocando en el mismo estrato la mecánica y la biomecánica. Las relaciones causales en procesos neuronales y extraneuronales son, por tanto, muy distintas. Así, desechan la idea de que, en casos reales, se extiendan procesos cognitivos al entorno. No intentan, en ningún caso, contra-

5 Clark, A. y Chalmers, D. (2011). La mente extendida. Oviedo, España: KRK Ediciones, pp. 18-19. 
decir un principio articulado por Clark y Chalmers, según el cual el mundo forma parte del proceso cognitivo. Pero lo que sin duda niegan es una extensión fáctica de lo cognitivo al mundo. Si tratamos de buscar la marca identificativa de los procesos propiamente cognitivos, observamos que se da sólo en los límites cerebrales. El transcranealismo, a su juicio, parece ignorar lo que se conoce hasta la fecha acerca del cerebro y el procesamiento cognitivo ${ }^{6}$. Clark responderá a esta réplica basándose en la noción funcionalista de los tipos explicativos comunes y unitarios de nivel superior que basan las relaciones causales. Así, se puede dar una homogeneidad general en las relaciones causales intracraneales y transcraneales sin olvidar una serie de relaciones causales subyacentes comprendidas en esa homogeneidad ${ }^{7}$.

Cabe destacar que Adams y Aizawa son funcionalistas, pero su crítica parte de que el funcionalismo no ampara la extensión que propone la TME. Ambos afirman que la cognición humana involucra procesos de contenido no derivado, esto es, los pensamientos que versan sobre objetos no requieren de convenciones externas. De esta manera, denotar a María con la palabra «María» se encontraría en el terreno de la convención lingüística, pero un pensamiento efectivo sobre María no requiere de ningún tipo de convención para poder hablar de ella. Por tanto, tan sólo los procesos neuronales codifican o implementan esos particulares de contenido no derivado, y por ello dichos procesos son los genuinamente cognitivos, frente a los extraneuronales, que incluirían particulares con contenido extrínseco o derivado, y por lo tanto no supondrían un verdadero proceso cognitivo. Así, los signos del bloc de notas al que hemos aludido carecen de contenido por sí solos; y aunque el sujeto lo consulte para lograr adquirir la creencia, no podemos decir que ese bloc forme parte del sistema cognitivo, ya que realmente lo que ahí se está produciendo es una codificación por parte del cerebro de toda la información relevante. Los procesos cognitivos se producen, así, tan sólo en el cerebro, excluyendo de un sistema cognitivo todos los componentes que no sean estrictamente neuronales ${ }^{8}$.

La marca de lo propiamente cognitivo es la base en el argumento de Adams y Aizawa para defender su intracranealismo contingente. Hay que partir del hecho de que ambos quieren diferenciar claramente entre la marca de lo mental y de lo cognitivo, por tanto dejan a un lado la cuestión de la conciencia para definir como lo propiamente cognitivo al aprendizaje, la percepción, la sensibilidad, la memoria o el pensamiento. Así, localizando la marca propia de los procesos cognitivos, es como podemos diferenciar claramente entre lo cognitivo y lo no cognitivo. Para ambos autores parece quedar claro que lo distintivo de los procesos cognitivos es propio de los límites cerebrales, de modo que los sistemas que enlazan cerebros con herramientas del entorno no parecen ser consecuentes con las bases de una ciencia

${ }^{6}$ Adams, F. y Aizawa, K. "The bounds of cognition», en Philosophical psychology, 14, 2001, pp. 43-64.

7 Clark, A. (2008). Supersizing the mind: Embodiment, action and cognitive extension. New York, EE. UU: Oxford University Press, p. 95.

8 Clark, A. y Chalmers, D. (2011). La mente extendida. Oviedo, España: KRK Ediciones, pp. 24-26. 
teórica. El hecho de que haya relaciones causales no implica que estemos hablando de un único sistema cognitivo. La apuesta de Adams y Aizawa no se basa en estas relaciones causales ni observa las similitudes con un sistema cognitivo para afirmar a partir de ahí que nos encontramos ante un sistema cognitivo propiamente dicho. Su apuesta es la de defender un modelo cognitivo fundamentado en los procesos físicos, químicos y biológicos?.

Robert Rupert también ha sido un importante crítico de la TME. Para él, la ciencia cognitiva y su metodología son incompatibles con una visión de la cognición extendida, ya que la propia ciencia busca un objeto de estudio persistente a lo largo del tiempo, y con el acoplamiento continuo de estructuras cognitivas adaptadas a dicho fenómeno se acaba perdiendo el sistema cognitivo único que se pretende estudiar. Lo que Rupert propondrá es la búsqueda de explicaciones más simples y conservadoras a las relaciones causales del sistema cognitivo, es decir, explicaciones que no superen la marca del sistema nervioso central; pues éstas serían afines a la explicación científica que precisa de la persistencia temporal de sistemas cognitivos. En otras palabras, la ciencia cognitiva es contraria a la cognición extendida, porque constitutivamente la ciencia precisa de fenómenos no sujetos al devenir propio de los sistemas acoplados de la TME ${ }^{10}$.

La defensa de Clark ante esta crítica será la afirmación de que el sistema nervioso central es el elemento más importante y activo de todo el sistema acoplado en el que se fundamenta la cognición. Así pues, dándole dicha importancia, niega que la hipótesis de una cognición extendida basada en interacciones causales con el entorno implique ningún tipo de rechazo del organismo como algo único y persistente. Por tanto, la ciencia cognitiva sigue teniendo cabida en un paradigma de vehículos cognitivos extendidos ${ }^{11}$. A Rupert, sin embargo, esta respuesta no le parece más que el fracaso de la cognición extendida y la decantación de Clark a favor de una visión tradicional de la cognición como algo interno al sistema nervioso central, sobre la cual aplica la etiqueta de 'extendida' 12 .

\section{EL ENIGMA DE LA CONCIENCIA}

No cabe duda de que la conciencia es algo tremendamente misterioso: «[No sabemos cómo] un cerebro, (o cualquier elemento físico) puede llegar a ser un lugar de experiencia consciente. Este será, seguramente, uno de los misterios metafísicos

9 Adams, F. y Aizawa, K. «The bounds of cognition», en Philosophical psychology, 14, 2001, pp. 62-63.

${ }_{10}$ Rupert, R. (2009). Cognitive systems and the extended mind. New York, EE. UU.: Oxford University Press, p. 60.

${ }^{11}$ Clark, A. (2008). Supersizing the mind: Embodiment, action and cognitive extension. New York, EE. UU: Oxford University Press, p. 139.

12 Rupert, R. (2009). Cognitive systems and the extended mind. New York, EE. UU.: Oxford University Press, p. 47. 
finales y no apuesto por que alguien pueda resolverlo» ${ }^{13}$. «No es que sepamos qué explicaría la conciencia y estemos teniendo problemas en encontrar una evidencia para seleccionar una explicación sobre otra; más bien es que no sabemos ni siquiera cómo debería ser una explicación de la conciencia» ${ }^{14}$.

La noción de conciencia que tratamos en este trabajo tiene que ver con la fenomenología de los estados mentales. Los estados mentales, en su aspecto fenomenológico, están ligados a la sensación o experiencia subjetiva que el propio individuo tiene de éstos. Un determinado estado mental puede tener unas concretas propiedades cuantitativas; por ejemplo, el dolor puede, en efecto, reducirse al disparo de las fibras 'C'. Sin embargo, lo exclusivo de la conciencia es cómo sentimos subjetivamente dicho estado mental; hablamos así de que ser conscientes de un estado fenomenológico, tal como el dolor, quiere decir que lo experimentamos. Así, también podemos expresar cuantitativamente que el color rojo es la percepción de una luz cuya longitud de onda dominante mide entre 618 y 780 nanómetros; y estaríamos hablando de un aspecto cognitivo del rojo. Sin embargo, lo que la conciencia percibe es la rojez del rojo; que aquí consideramos como el aspecto fenomenológico de dicho estado mental.

La aporía viene dada por la insuficiencia explicativa a la hora de mostrar que un evento cualitativamente subjetivo equivale a algo material y cuantitativo. Joseph Levine nos muestra el abismo explicativo que hay entre el enunciado paradigmático de los materialistas 'el dolor es el disparo de las fibras $\mathrm{C}^{\prime}$ y un estado mental subjetivo tal como el dolor en sí mismo ${ }^{15}$. La cuestión fundamental es, pues, ¿cómo podemos superar el abismo que separa lo objetivo y lo subjetivo, es decir, el cerebro y la experiencia consciente? Nagel afirmará que las perspectivas perceptivas son algo propio, no del sujeto, sino del aparato perceptivo de cada especie. La ciencia describe los fenómenos, pero les quita su carácter fenomenológico a través de la teorización. Es decir, podemos definir con la tesis de la identidad un color como un evento cerebral, pero lo que la experiencia del color constituye no se puede transmitir mediante la ciencia. Del mismo modo, entre las distintas especies no podemos compartir esas perspectivas de conciencia, porque, al fin y al cabo, jamás sabremos cómo concibe el mundo un murciélago más allá de lo explicativo ${ }^{16}$.

13 Fodor, J. (1998). In Critical Condition: Polemical Essays on Cognitive Science and the Philosophy of Mind. Cambridge, MA: MIT Press,p. 83.

${ }_{14}$ McGinn, C. (1999). The Mysterious Flame: Conscious Minds in a Material World. New York, EE. UU.: Basic Books, p. 61.

${ }^{15}$ Levine, J. «Materialism and qualia», en Chalmers, D. (2002). Philosophy of mind: classical and contemporary readings. New York, EE. UU.: OUP, p. 357. p. 442 .

${ }^{16}$ Nagel, T. "What is it like to be a bat», en The Philosophical Review, vol. 83, n. ${ }^{\circ}$ 4, 1974, 


\section{LA CONCIENCIA ¿EXTENDIDA?}

Parece que se ha hecho evidente que para Clark y Chalmers los procesos cognitivos no están, al menos todos, en la cabeza. Incluso podríamos afirmar que ciertos estados mentales pueden extenderse también al entorno al necesitar componentes extraneuronales en un circuito hacia el éxito cognitivo, gracias a que los vehículos de la cognición pueden atravesar las fronteras impuestas por el cráneo. Sin embargo, llegamos a la cuestión problemática de la conciencia: ¿se extiende ésta también al entorno?

Ya en su artículo original de 1998 ambos parecen dar a entender que el hecho de que la conciencia sea también extendida no resulta tan claro, y que parece poco admisible que ésta supere los límites cerebrales. Así que Clark y Chalmers marcan la distinción entre procesos cognitivos y conciencia, afirmando que, mientras lo cognitivo sí se extiende, la conciencia se queda dentro de nuestro horizonte intracraneal. En su obra de 2009 Supersizing the mind, Clark ahonda con mayor precisión en el problema que presenta la conciencia en una concepción de la mente extendida. Afirmará que, en efecto, las creencias, los procesos cognitivos, los mecanismos de la percepción y los estados de ánimo se pueden posicionar como algo extensible más allá de la conciencia, y que precisamente es la parte no consciente de todos éstos la que llegaría a superar los límites neuronales.

En principio parece ser que no hay una razón por la que las bases físicas de la conciencia no puedan extenderse de una forma similar, incluso podríamos imaginar un mundo posible en el que el entorno sea un elemento directo de la conciencia. Sin embargo, en nuestro mundo, tal y como está constituido, no podemos afirmar, dadas las condiciones, que la conciencia se extienda del mismo modo que el proceso cognitivo del sujeto que accede a un recuerdo con su bloc de notas. Parte de la razón es que las bases físicas de la consciencia requieren de acceso directo a la información en una sensibilidad extremadamente detallada con la información del entorno, así como un gran ancho de banda y una sincronización nanométrica, y dicha posibilidad sólo se da en el propio aparato neuronal, dentro del sistema nervioso central. La conexión consciente con el entorno sería mucho más lenta, y no permitiría la conciencia ${ }^{17}$.

Lo que está claro es que, en su obra de 2009, «Spreading the Joy? Why the Machinery of Consciousness is (Probably) Still in the Head", expone de nuevo por qué la maquinaria de la conciencia sigue permaneciendo en los límites neuronales y no se extiende como el resto de la mente. Pero, antes de dar de lleno con la propuesta que argumentaría el porqué de esa conciencia 'contenida' en los límites del sistema nervioso central, lo que Clark hará será mostrar con precisión los distintos puntos de apoyo que se han ido proponiendo en filosofía de la mente a una conciencia extendida, negándolos todos a la postre. Su pretensión es demostrar que nin-

17 Clark, A. (2008). Supersizing the mind: Embodiment, action and cognitive extension. New York, EE. UU: Oxford University Press. Foreword xIv-xv. 
gún argumento usado en la fundamentación de la tesis de una cognición extendida puede llevarnos a la aceptación de una conciencia extendida ${ }^{18}$.

\subsection{El ARgumento ENACTIVISTA}

Uno de los mayores defensores de una conciencia extendida es Alva Noë, externalista y enactivista. El enactivismo viene a dar cuenta del importantísimo papel de la actividad que el organismo genera con el entorno en la construcción del sujeto en toda su amplitud. Así, Noë presenta una postura muy convincente y defendible, a saber: que el sujeto no se constituye por sí solo. Ahora bien, esta visión externalista extrema incluiría algo muy importante que generará el rechazo de Clark: que el carácter y el contenido de la experiencia perceptual serían también constituidos por ese entorno. La propuesta enactivista mantiene que la percepción no es algo que sucede en nosotros o se nos presenta, es algo que nosotros hacemos. De este modo, "la experiencia perceptiva adquiere contenidos gracias a nuestra posesión de habilidades corporales. Lo que percibimos es determinado por lo que hacemos (o por lo que sabemos cómo hacer); está determinado por lo que estamos preparados para hacer. Nuestra experiencia perceptiva es enactiva: la activamos fuera ${ }^{19}$. Por tanto, la conciencia y el contenido de los estados mentales estarían determinados por un proceso práctico-operatorio que el propio agente ejecuta con el entorno usando los denominados bucles sensoriomotores, circuitos que enlazan el cerebro con el entorno de modo similar a la retroalimentación que Clark y Chalmers proponían en su artículo original.

La propuesta enactivista está respaldada por numerosos estudios y experimentos. En 2003, Bach y Rita desarrollaron un sistema de sustitución visual. El estudio consistía en colocar a unos sujetos ciegos unos mecanismos con cámaras que convertían la información visual en pequeños estímulos eléctricos que alteraban según la distancia, color, forma, superficie y otra serie de variables. Así los sujetos lograban saber ante lo que se encontraban gracias a dichos estímulos. La cuestión importante es que, al cabo de un tiempo, los propios individuos ciegos afirmaban que las sensaciones táctiles se difuminaban para ser reemplazadas por estímulos cuasivisuales, que les permitían incluso comportamientos tales como el reflejo de esquivar una pelota que les era lanzada a la cabeza. Por tanto, para los enactivistas es importante aprender de los nuevos bucles sensoriomotores. Sólo cuando el sujeto comienza a aprender acerca de los modos activos y práctico-operatorios con el entorno llevando consigo esa cámara, entonces la experiencia comienza a ser cuasivisual. Este resultado lleva a Noë a afirmar rotundamente que lo que determina la propia experiencia consciente no es la actividad neuronal, sino el modo en

${ }^{18}$ Clark, A. «Spreading the Joy? Why the Machinery of Consciousness is (Probably) Still in the Head", en Mind, 118, 2009, pp. 968.

19 NoË, A. (2004). Action in Perception. Cambridge, MA: MIT Press, p. 1. 
el que esa actividad neuronal está acoplada en la dinámica sensoriomotora, el propio hecho de que se encuentre 'acoplada' en el circuito que conecta con el entorno es lo que hace que la conciencia esté extendida ${ }^{20}$.

«La aproximación enactiva busca explicar la cualidad de la experiencia perceptiva, no como una función neuronal causada por procesos en el cerebro... sino más bien en términos de patrones y estructuras de la actividad práctica. En la aproximación enactiva el cerebro, el cuerpo y el mundo trabajan juntos para hacer posible la conciencia [...]. La experiencia no está causada por el cerebro, ni se realiza en ella. La experiencia se realiza en la vida activa del animal hábil (práctico)»»21.

\subsection{El ARgUMENTO DE LA EXPERIENCIA VISUAL}

Existe otro argumento de corte enactivista que defiende la extensión de la conciencia al entorno, según el cual la experiencia visual está parcialmente constituida por nuestras acciones, es decir, nuestros movimientos corporales, guiños, giros, etc., que en su conjunto forman parte de riqueza de la experiencia visual. La mejor explicación del detalle de los fenómenos que percibimos visualmente es que dichas experiencias, según los requisitos de la TCE, no están determinadas por la actividad neuronal solamente.

Podríamos admitir que el detalle o nitidez propia de la visión es fruto de las células sensitivas que capturan el color, que están, casi todas, en una densa región fotorreceptora de alta resolución que se aloja en la fóvea -un área de la retina que se encarga de enfocar los rayos luminosos que dan lugar al color-. Así, en última instancia el detalle de nuestra experiencia perceptiva estará determinado por la codificación de los datos producidos por esta zona en el cerebro. Pero para Nöe: «El guiño del ojo, el giro de la cabeza, el movimiento del cuerpo... todos ellos nos dan el detalle que necesitamos tal y como lo necesitamos. El mundo está presente virtualmente gracias a nuestros accesos dinámicos en línea» ${ }^{22}$.

\subsection{El argumento del entrelaZAmiento dinÁmico}

El llamado entrelazamiento dinámico es otro argumento en defensa de la conciencia extendida que Clark analiza y rechaza. Es defendido por Thompson, Varela y Cosmelli principalmente, y surge de la concepción funcionalista relacionada con los bucles; pero yendo más allá, afirmando que hay que dejar de pensar en términos de un flujo simple y lineal, en el cual los sentidos entregan un 'input'

${ }^{20}$ Clark, A. «Spreading the Joy? Why the Machinery of Consciousness is (Probably) Still in the Head", en Mind, 118, 2009, pp. 969-970.

${ }^{21}$ Nö̈, A. (2004). Action in Perception. Cambridge, MA: MIT Press, p. 227.

22 Nö̈, A. (2006). "Experience without the head», en Gendler, T. y Hawthorne, J. (2006). Perceptual experience. Oxford, U.K.: Oxford University Press, p. 420. 
que es procesado hasta llegar al 'output'. Encontramos, por tanto, una alternativa al modelo clásico funcionalista que ya conocemos, en el que la dinámica de bucles y procesamiento es mucho más rica: la información fluye hacia atrás, hacia arriba y más o menos continuamente. De este modo, los vehículos físicos son, en ciertas ocasiones, extendidos dinámicamente en bucles que conectan las áreas superiores del cerebro con las inferiores, y las rodean de nuevo, logrando la unión de los sistemas cognitivo y motor. La cuestión es llevar esto a un argumento a favor de la TCE; así, Thompson y Cosmelli ańadirán que la contribución del cuerpo no neuronal es tan importante y está tan complejamente entretejida con el procesamiento neuronal en sí mismo que no podemos simplemente quedarnos con los elementos neuronales; dejando de un lado al resto y separándolos. Su pretensión es «subrayar la inmensa complejidad de las interacciones neuronales y extraneuronales que en última instancia determina la actividad cerebral en un organismo vivo» ${ }^{23}$.

Para Varela y Thompson las experiencias de la emoción, por tomar un posible ejemplo, dependen de un complejo proceso de bucles entre el cerebro y el mundo extraneuronal extendido en el tiempo. Supongamos que rechazamos, al igual que Noë, lo que él llamaba 'la imagen instantánea de una experiencia visual', como algo totalmente determinado por los estados cerebrales en un momento específico del tiempo; y en su lugar sugerimos que estos procesos necesarios para la experiencia de conciencia evolucionan esencialmente en el tiempo (lo cual hemos mencionado en el argumento anterior, luego la clave es una vez más la extensión temporal de una experiencia fenoménica como fundamentación de que el internismo no es una propuesta válida).

\subsection{El RECHAZO DE LA CONCIENCIA EXTENDIDA}

¿Cómo muestra Clark la insuficiencia argumentativa de lo que propone el enactivismo? La explicación de Nöe no hace más que mostrar una evidencia en el explanandum según la cual los parámetros neuronales han sido modificados gracias al ejercicio práctico con el entorno; luego el bucle sensoriomotor ha tenido éxito. Sin embargo, partiendo de eso, no podemos hablar de que el bucle sensoriomotor sea el que genere experiencia consciente. Lo único que nos puede aportar la propuesta enactivista es la evidencia de que hay un bucle que se forma entre entorno y organismo, y que sirve como condición previa para lograr una modelación neuronal. Pero, para Clark, es esa actividad neuronal misma la que genera la experiencia consciente; precondicionada, sí, por el bucle con el entorno, pero, al fin y al cabo, producida en el cerebro, y no en el acoplamiento en el bucle. Clark admite el bucle sensoriomotor, lo que no admite es que la conciencia se encuentre acoplada en el

${ }^{23}$ Clark, A. «Spreading the Joy? Why the Machinery of Consciousness is (Probably) Still in the Head", en Mind, 118, 2009, pp. 976. 
bucle. Por ello, permite cierta relación de la conciencia con el entorno; pero no hasta el punto de hablar de vehículos de la conciencia.

Todo esto no quiere decir que la TME no comparta con la visión enactivista el absoluto rechazo a la suficiencia neuronal. Pero dicho rechazo no puede estar justificado en las bases del sistema de sustitución visual o los demás estudios registrados en respaldo del enactivismo. Los estudios sólo muestran el último eslabón de una cadena explicativa; tan sólo presentan la evidencia de que el cerebro ha sido modelado; pero en última instancia la conciencia sigue perteneciendo al reino intracraneal ${ }^{24}$.

Los motivos que llevan al rechazo del segundo y el tercer argumento a favor de una conciencia extendida por parte de Clark son los mismos, y parten de que para afirmar que un movimiento forma parte del contenido de la experiencia visual debemos hablar de potencialidades, es decir, la experiencia no está constituida en el momento, sino más bien se irá constituyendo según «nos movamos». La defensa de la TCE llevaría a admitir que lo que fija una experiencia no es un momento determinado de la actividad neuronal, sino procesos extendidos en el tiempo. Pero admitir esto no sería sino admitir, una vez más, que la maquinaria de la conciencia está en la cabeza; añadiendo solamente que ésta sigue un determinado camino de evolución. Por esto Clark no defiende esta tesis como suficientemente robusta ${ }^{25}$. Si la tesis del entrelazamiento dinámico como defensa de la TCE en última instancia también se fundamenta en la llamada extensión temporal de los fenómenos, entonces la crítica y legitimación de dicho argumento por parte de Clark será igual que la anterior: el hecho de que los estados neuronales precisen de una evolución a lo largo del tiempo para fundamentar una experiencia consciente no niega la explicación internista.

\subsection{LA CONCIENCIA EN LA CABEZA}

Hasta el momento parece haber quedado claro que para Clark la defensa de una cognición extendida no implica necesariamente que la experiencia consciente supere también los límites marcados por el sistema nervioso central. Así, los argumentos de corte enactivista han sido negados o considerados inconcluyentes para soportar la consideración de una conciencia extendida. Como hemos afirmado, Clark mantiene la postura de una conciencia imbuida en los límites cerebrales. A continuación, mostraremos los argumentos que sustentan esta concepción (que ya hemos dibujado brevemente), y seguidamente en la conclusión abarcaremos las posibles tensiones que pueden llegar a generar.

El filósofo y neurocientífico Chris Eliasmith nos sugiere que la dinámica interna en el cerebro es 'cualitativamente diferente' de la que cubre el límite del cerebro-mundo, y que las diferencias clave incluyen la velocidad y el ancho de banda.

\footnotetext{
${ }^{24}$ Ibid., pp. 970-971.

${ }^{25}$ Ibid., pp. 974-975.
} 
Una vez más vemos que el gran problema que basa la imposibilidad de una mente totalmente extendida al entorno es la incapacidad de que los vehículos entre cerebro y entorno puedan lograr la velocidad necesaria y la sincronización requerida por la conciencia ${ }^{26}$.

«Las diferencias más obvias [entre las dinámicas neuronales y supraneuronales] son la velocidad del flujo de información (o ancho de banda), y el grado y tipo de acoplamiento. Puesto que los cuerpos tienen masa, tienden a ralentizar la transferencia de información hacia el resto del mundo desde el cerebro (en efecto, actúan como un filtro de paso lento). Sin embargo, no existe ningún impedimento en el flujo de información dentro de las áreas del cerebro. Esto es resultado de una enorme diferencia entre el tipo de acoplamiento que puede ser soportado entre los subsistemas del cerebro y entre el cerebro y el entorno externo.... ${ }^{27}$.

La consideración crucial es que la experiencia consciente es una materia de alta velocidad y con una sincronización temporal muy fina, y los vehículos materiales deben estar integrados unos con otros a dicha velocidad, cosa que sólo es posible en las estructuras del cerebro ${ }^{28}$. La sincronización requerida para el proceso de la conciencia debe ser extremadamente precisa, y en los vehículos que sustentan la comunicación cognitiva entre sistema nervioso central y entorno se pierde esa precisión. Todo esto nos aboca a aceptar que los circuitos cognitivos deban llevarse a cabo dentro del propio sujeto y, más concretamente, en sus estructuras neuronales.

El problema viene dado por una contradicción que pone en tela de juicio la validez de toda la TME; y es que, si basamos toda nuestra teoría de los vehículos de cognición y estados mentales en el carácter funcional y de sentido común de los vehículos (en las similitudes de grano grueso), no podemos aludir a los detalles neurofisiológicos más precisos (de grano fino) como fundamento de que la conciencia no esté extendida. En otras palabras: si defendemos las consideraciones de grano fino no podemos argumentar que los vehículos cognitivos se extiendan al entorno; puesto que el grano grueso o sentido común eran el sostén de la paridad funcional, y si defendemos las consideraciones de grano grueso, no podemos negar que la conciencia se extienda mediante argumentos del tipo de la sincronización y precisión neuronal. Defender grano grueso en el ámbito de la cognición y grano fino en el de la conciencia nos lleva a una tensión; en la que, al parecer, o negamos la mente extendida o afirmamos la conciencia extendida.

Según este prisma, la defensa de una cognición extendida es un reclamo no fisicalista basado en la paridad funcional, pero la conciencia sí sería, sin embargo, reducible materialmente. Tenemos pues, que dentro de la misma noción trabajamos con dos convicciones distintas y casi contradictorias sobre una misma realidad -la mente-, cosa que se muestra, como poco, aporética. Defender la TME implica-

26 Ibid., pp. 983-984.

27 Eliasmith, C. (2008). Dynamics, Control, and Cognition. Cambridge, UK: Aydede and Robbins, p. 150.

28 García Rodriguez, A. "An Extended View of Mind and Cognition», en Teorema, vol. 30/2, 2011, p. 9. 
ría trabajar con realidades o entidades distintas, afirmando que la mente es a la vez física y funcional. El sentido común, sin embargo, nos lleva a pensar que, o todas las entidades correspondientes a la mente son pertenecientes a una misma categoría, o de lo que estamos hablando es de entidades distintas.

Por otro lado, la apelación constante a elementos intracraneales que expliquen la génesis de la conciencia por parte de Clark se topa con el problema consustancial a toda propuesta fisicista, en la medida en que es incapaz de resolver cómo un evento cualitativamente subjetivo equivale a algo material y cuantitativo. En este sentido, no es capaz de solventar la aporía del salto que se da de lo neuronal a lo experiencial. Resulta paradójico, pues, que el funcionalismo surgiese en sus orígenes como una teoría cuya pretensión era dar una interpretación comprensible a los abismos explicativos con los que se topaban las tesis materialistas, y que, sin embargo, la TME, nacida en el seno del funcionalismo, acabe invocando a las bases físicas del cerebro como argumento para dar cuenta de la conciencia.

ReCIBIDO: noviembre 2019; ACEPTADO: diciembre 2019 
